\title{
Sistemática para Análise de Séries de Tráfego de Rede Sob o Espectro da Teoria do Caos
}

\author{
Évelynn Regina da Costa Rosa e Flávio Geraldo Coelho Rocha
}

\begin{abstract}
Resumo-Esse artigo sistematiza um algoritmo de análise de séries temporais, neste caso de tráfego de redes de comunicações, desenvolvido com o propósito de quantificar parâmetros referentes à Teoria do Caos, descrevendo um procedimento sequencial padrão de análise e medida de dados experimentais que apresentam características não-lineares, dentro do espectro da Teoria do Caos e seus procedimentos seminais. Reconstruir o atrator, determinar dimensão de correlação e dimensão de incorporação, estimar o Expoente de Lyapunov e o Parâmetro de Hurst são os passos seguidos nessa análise.
\end{abstract}

Palavras-Chave-Caos, Tráfego de Rede, Dimensão de Correlação, Reconstrução do Espaço de Fase, Expoente de Lyapunov, Parâmetro de Hurst

Abstract-This paper systematizes an analysis algorithm for time series, from network traffic in this case, developed with the purpose of quantifying Chaos Theory parameters, thus describing a standard sequential procedure to analyze and classify experimental data which displays non-linear characteristics, within the spectrum of Chaos Theory and its seminal procedures. Reconstructing the attractor, determining embedding delay and correlation dimension, estimating the Lyapunov Exponent and the Hurst Parameter are the steps followed in this analysis.

Keywords-Chaos, Network Traffic, Correlation Dimension, Phase-Space Reconstruction, Lyapunov Exponent, Hurst Parameter.

\section{INTRODUÇÃO}

A teoria do caos descreve uma variedade de sistemas nãolineares e por isso já foi abordada nas mais diversas áreas como, por exemplo, a meteorologia [20], a química e filosofia [27], o jornalismo [10], a física [33], a matemática [23], a construção de reservatórios [13], a previsão do tempo [19], os ciclos pluviais e a bolsa de valores [21], [22], [3], [1], a topologia [36], e a coleta e processamento de dados [38], [7], [29], [18], [2], [37].

O comportamento das redes de dados apresenta, de forma geral, características que possibilitam sua análise de acordo com essa abordagem não-linear adotada pela Teoria do Caos, de forma que possam ser analisadas através de alguns parâmetros específicos como a dimensão de correlação, o Expoente de Lyapunov, e o Parâmetro de Hurst, como já foi discutido em diversos trabalhos seminais [35], [24], [12], [17], [15].

Nas redes de dados, o interesse reside na captura de características fundamentais de comportamento do sistema a fim de garantir a qualidade de serviço através do monitoramento de métricas tais como banda, atraso, probabilidade de perda de pacotes e taxa de alocação [32], [28]. Desse modo, para que

Évelynn R. Rosa e Flávio G. C. Rocha, Escola de Engenharia Elétrica Mecânica e Computação. Universidade Federal de Goiás, Goiânia-GO, Brasil e-mail: wltnschng@gmail.com e flaviogcr@ufg.br. metas de qualidade de serviço sejam alcançadas, é necessário que se entendam as características do tráfego de forma que seja possível desenvolver uma modelagem precisa, possibilitando seu planejamento, melhoramento e manutenção [7].

Portanto, este trabalho sistematiza um algoritmo de quantificação e parametrização de séries temporais caóticas utilizando ferramentas focadas em sistemas dinâmicos. O restante desse trabalho está organizado da seguinte maneira: na Seção II descrevem-se as três abordagens de análise: a reconstrução do espaço de fase com o auxílio do método da autocorrelação, e os cálculos do Expoente de Lyapunov e do Parâmetro de Hurst. Os experimentos realizados nas séries temporais estão descritos na Seção III, enquanto na Seção IV estão as conclusões.

\section{Medidas de ClassificaÇão do Tráfego com Base NA TEORIA DO CAOS}

O primeiro passo nessa análise de comportamento das séries temporais é a reconstrução do espaço de fase. Ao longo deste processo serão descritas grandezas através das quais aspectos comportamentais do sistema serão quantificados, tais como dimensão (através da dimensão de correlação e da dimensão de incorporação), e correlação (através do atraso ideal calculado e do parâmetro de Hurst), descritos a seguir.

\section{A. Reconstrução do Espaço de Fase}

A reconstrução do espaço de fase tem o propósito de embutir uma série univariada em um espaço multidimensional de modo que se possam representar suas dinâmicas subjacentes [26]. Essa reconstrução é feita através do uso de uma variável de atraso de tempo, $\tau$, eliminando amostras redundantes a fim de construir um vetor atraso, e a trajetória reconstruída $\mathbf{X}$ pode ser expressa como uma matriz na qual cada linha é um vetor espacial $\mathbf{x} \in \Re^{m}$ [14], [7].

Definamos um vetor bidimensional [33]

$$
\mathbf{x}(t)=(B(t), B(t+\tau)),
$$

para um atraso $\tau>0$, de forma que a série temporal $B(t)$ gere uma trajetória $\mathbf{x}(t)$ em um espaço de fase bidimensional. Se definirmos um vetor tridimensional [33]

$$
\mathbf{x}(t)=(B(t), B(t+\tau), B(t+2 \tau)),
$$

obteremos um espaço de fase tridimensional, e assim sucessivamente [33].

Uma série temporal representa, então, uma sequência escalar de medidas de alguma quantidade que reflete o estado do 
sistema no instante de medida, coletadas em múltiplos de uma amostragem temporal fixa [14]:

$$
x_{n}=x(\mathbf{s}(n \Delta t))+\eta_{n},
$$

na qual s é a função de medida e $\eta_{n}$ é o ruído inerente à medida. Uma reconstrução por atrasos em $m$ dimensões, desconsiderando o ruído $\eta_{n}$, é então constituída pelos vetores $\boldsymbol{X}_{n}$, construídos da seguinte maneira [30], [7], [18]:

$$
\boldsymbol{X}_{n}=\left(x_{i}, x_{i+\tau}, \ldots, x_{i+(m-1) \tau}\right),
$$

na qual $\tau$ é o atraso de reconstrução, $m$ é a dimensão de incorporação, e $\boldsymbol{X}_{n}$ é o vetor $m$-dimensional. A trajetória reconstruída $\mathbf{X}$ pode então ser expressa como uma matriz na qual cada linha $\boldsymbol{X}_{n}$ é um vetor estado de fase de $m$ atrasos, ou seja [30], [7], [18]:

$$
\mathbf{X}=\left(\boldsymbol{X}_{1}, \boldsymbol{X}_{2}, \ldots, \boldsymbol{X}_{N}\right)^{T}
$$

A diferença espacial entre as amostras $\tau$ (ou em unidade de tempo $\tau \Delta t$ [14] entre componentes adjacentes dos vetores atraso) é o atraso em si, e o atrator formado por $\boldsymbol{X}_{n}$ será equivalente ao atrator no espaço desconhecido ao qual o sistema original pertence se a dimensão $m$ do espaço de coordenadas de atraso for suficientemente alta.

$\mathbf{X}$ será então uma matriz $N \times m$, e as constantes $N, n, m$ e $\tau$ se relacionam da seguinte maneira [30], [7], [18]:

$$
N=n-(m-1) \tau \text {. }
$$

Portanto, a reconstrução do espaço de fase requer o cálculo de variáveis que determinam sua estrutura (Equação 6), como a dimensão de incorporação $m$, tratada na Seção II-D. Ela é escolhida de forma que $m>2 D_{2}+1$, na qual $D_{2}$ é a dimensão de correlação (Seção II-C). Se todos esses requisitos forem cumpridos, então o espaço de fase reconstruído é equivalente ao original, obedecendo o princípio do difeomorfismo [24], [35].

\section{B. $O$ atraso $\tau$}

A distância espacial entre as posições das medidas é denominada de atraso $\tau$, como mostram as Equações 1, 2, e 4, e seu valor apropriado é usado na reconstrução do espaço de fase, havendo, portanto, na escolha desse valor, uma negociação entre redundância e decorrelação entre as amostras da série temporal: a meta é diminuir a redundância enquanto se preserva a correlação, uma vez que quanto menor a distância entre as amostras mais elas tendem a estar correlacionadas. Por outro lado, elas podem parecer completamente não-correlacionadas se estiverem muito distantes entre si [14].

O método da autocorrelação para o cálculo de $\tau$ define que o atraso ideal é obtido de forma que a função de autocorrelação atinja o valor de $1-1 / e,(e=2.7183)$ [30], [7].

\section{Dimensão de Correlação}

A dimensão de correlação $D_{2}$, inicialmente formulada em [11] e [12], descreve como o objeto geométrico de interesse deve ser reconstruído a partir de uma amostra finita de dados pontuais [30], [7], [14], [18]:

$$
D_{2}=\lim _{r \rightarrow 0} \frac{\partial \ln C_{m}(r)}{\partial \ln (r)},
$$

na qual $C_{m}(r)$ é a integral de correlação que deve ser calculada da seguinte maneira [30], [7], [14], [18]:

$$
C_{m}(r) \approx \frac{2}{N(N-1)} \sum_{i=1}^{N} \sum_{j=i+1}^{N} \Theta\left(r-\left\|\mathbf{x}_{i}-\mathbf{x}_{j}\right\|\right),
$$

na qual $\Theta$ é a função degrau de Heaviside, que estipula que $\Theta(x)=0$ se $x \leq 0$ e $\Theta(x)=1$ se $x>0$. O somatório conta os pares $\left(\mathbf{x}_{i}, \mathbf{x}_{j}\right)$ cuja distância entre si é menor que $r$, e $\left\|\mathbf{x}_{i}-\mathbf{x}_{j}\right\|$ é a distância entre os vetores $Y_{i}$ e $Y_{j}$, definida como [30], [7], [14], [18]:

$$
\left\|\mathbf{x}_{i}-\mathbf{x}_{j}\right\|=\max \left\{\left|x_{i+l \tau}-x_{j+l \tau}\right|: 0 \leq l \leq m-1\right\},
$$

e $D_{2}$ é , então, a inclinação da reta [30], [7], [14], [18]:

$$
D_{2}=\frac{d \ln C_{m}(r)}{d \ln (r)}
$$

ou seja, uma vez que os vetores de incorporação $\mathbf{X}_{n}$ são reconstruídos, a estimativa da dimensão de correlação poderá ser feita em dois passos: primeiramente deve-se calcular o somatório da correlação definida pela Equação 8, na faixa estipulada para $r$ e para várias dimensões de incorporação $m$, para então se inspecionar $C(r)$ a fim de verificar a presença de autossimilaridade: se for satisfatória, pode-se subsequentemente calcular um valor para a dimensão [30], [7], [14], [18].

Ao se plotar um gráfico de $D_{2}$ para cada valor de $m$ surgirá uma região plana, para um $m$ suficientemente alto, que será o indício da estabilização do valor de $D_{2}$. O intervalo da inclinação da reta durante o qual a curva é aproximadamente linear é chamado de região de escala, a partir da qual o expoente de Lyapunov é calculado, como descreve a Seção II-E [14].

\section{Dimensão de Incorporação - $m$}

A dimensão de incorporação $m$ diz respeito à quantidade de medidas nos vetores atraso a serem reconstruídos na matriz de reconstrução do espaço de fase [14], [34]. Tecnicamente, é preciso que existam tantos atrasos quanto necessários para que o atrator subjacente possa ser nitidamente visualizado no espaço de fase, e por isso o procedimento usual é aumentar a dimensão de incorporação $m$ a cada iteração e assim calcular as dimensões de correlação $D_{2}$ dos atratores resultantes. Os valores aumentarão sucessivamente até que a dimensão de incorporação atinja um valor alto o bastante, permitindo assim que o atrator e o valor estimado da dimensão de correlação $D_{2}$ se estabilizem [34].

\section{E. O Expoente de Lyapunov}

Nos sistemas periódicos as trajetórias predominantemente divergem lentamente ao longo do tempo, mas nos sistemas caóticos essa separação é exponencialmente rápida. $\mathrm{O}$ valor médio desse aumento, o expoente de Lyapunov, é característico de cada sistema, e quantifica a intensidade do caos ao 
caracterizar a divergência das trajetórias à medida que o fluxo caótico se propaga [14], [4].

Um algoritmo eficaz no cálculo do expoente de Lyapunov, usado neste trabalho, é o de Michael T. Rosenstein [30], conhecido como Small Data Sets, cujo fluxograma se encontra sintetizado em [7].

\section{F. O Parâmetro de Hurst}

O parâmetro de Hurst, primeiro formulado em [13], é usado para classificar séries temporais quanto ao seu comportamento de persistência ou memória. Sua importância está vinculada ao fato de o desempenho das redes estar diretamente relacionado à frequência de rajadas no tráfego, as quais, por sua vez, estão relacionadas ao valor do parâmetro de Hurst, medida frequentemente utilizada para medir o comportamento fractal. $\mathrm{O}$ valor de $\mathrm{H}$ pode ser estimado a partir do decaimento da função de autocovariância normalizada (autocorrelação) e está relacionado ao comportamento da densidade espectral de potência em baixas frequências. $\mathrm{O}$ valor de $\mathrm{H}$ pode ser usado na análise do tráfego de rede para determinar ou predizer o comportamento em rajadas, o que pode impactar diretamente o desempenho da rede em análise [9]. Com valores distribuídos entre o intervalo $0<H<1$, seus subintervalos são classificados da seguinte maneira:

- $\mathbf{H}<\mathbf{0 , 5}$ - as medidas tendem a alternar entre valores baixos e valores altos (periodicidade);

- $\mathbf{H} \approx \mathbf{0 , 5}$ - as medidas tendem a apresentar valores com uma distribuição estocástica (aleatória);

- $\mathbf{H}>\mathbf{0 , 5}$ - valores altos tendem a ser seguidos por outros valores altos; valores baixos tendem a ser seguidos por valores baixos e essa tendência se mantêm num futuro infinito (persistência de longa duração) [13], [21].

Um fluxograma do algoritmo está ilustrado na Fig.1.

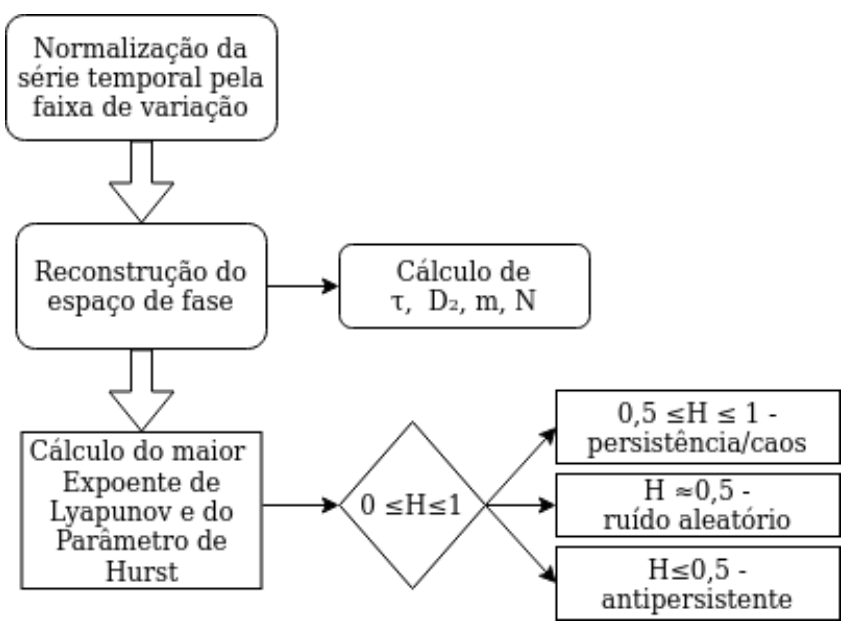

Fig. 1. Fluxograma

\section{EXPERIMENTOS DE ANÁlise DAS SÉRIES TEMPORAIS}

Os resultados experimentais encontrados nas análises das séries reais de tráfego de rede estão descritos a seguir, e os intervalos utilizados nessa análise, para cada série, estão organizados na Tabela I, cujas respectivas 5000 amostras estão ilustradas na Fig. 2.

As séries InterBC89 e InterDEC2 (cujos nomes originais são BCpAug89 e dec-pkt-2, respectivamente) são de tráfego Ethernet (da Bellcore Morristown Research and Engineering [6]) e a série InterMultipathTCP (nome original MultipathTCP) representa o tráfego de uma rede sem fio (wireless) que utiliza o protocolo Multipath TCP [5], que é uma extensão do protocolo de controle de transmissão ( TCP - Transmission Control Protocol). Estas séries foram escolhidas por estarem amplamente presentes na literatura ([16], [28], [31]), possibilitando a comparação entre resultados [37].

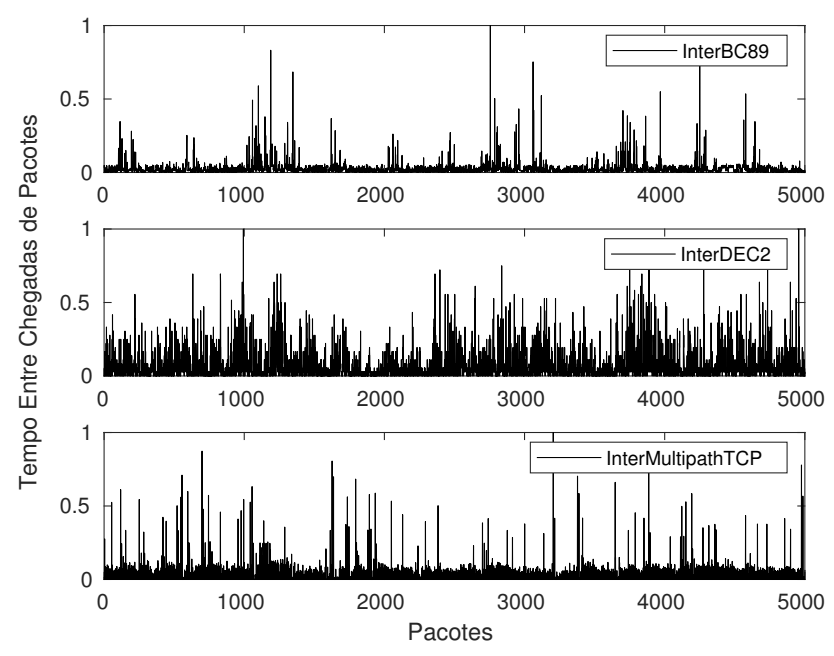

Fig. 2. Séries Temporais

Ficam evidentes, nos gráficos das três séries, a presença de rajadas, cujas amplitudes se mostram bastante elevadas em relação à media, principalmente para as séries InterBC89 e InterMultipathTCP (Fig. 2). Já os gráficos das integrais de correlação, ilustrados nas figuras 3, 4 e 5, foram computados com o uso do algoritmo de Grassberger-Procaccia [11], [12], para espaços de fase reconstruídos, com dimensões de incorporação variando entre $m=2$ e $m=16$ [7].

A relação entre $C_{m}(r)$ e $r$ indica regiões de escala, nas séries das figuras 3 e 4 , para todas as dimensões de incorporação $(m)$ usadas, evidenciadas no afunilamento das retas, sinal de uma lei de potência (power law) presente, permitindo assim estimativas relativamente precisas das dimensões de correlação $D_{2}$ [14]. A série da Fig. 5 apresenta retas quase paralelas, indicando ausência de uma lei de potência.

A Fig. 6 mostra a dimensão de correlação $D_{2}$ em relação à dimensão de incorporação $m$ para as três séries analisadas, e os valores de $\tau$ e $D_{2}$ encontrados estão organizados na Tabela I, na qual se observa que a série InterDEC2 possui maior dimensionalidade entre todas as séries analisadas.

O expoente de Lyapunov $(\lambda)$ está relacionado à dependência sensível às condições iniciais: ele quantifica o grau de expansão ou contração das órbitas do atrator, delimitando um horizonte temporal além do qual previsões aceitáveis se tornam impossíveis [34]. Observando a Fig. 7 pode-se perceber qual a faixa de valores necessária para seu cálculo, que está associado 


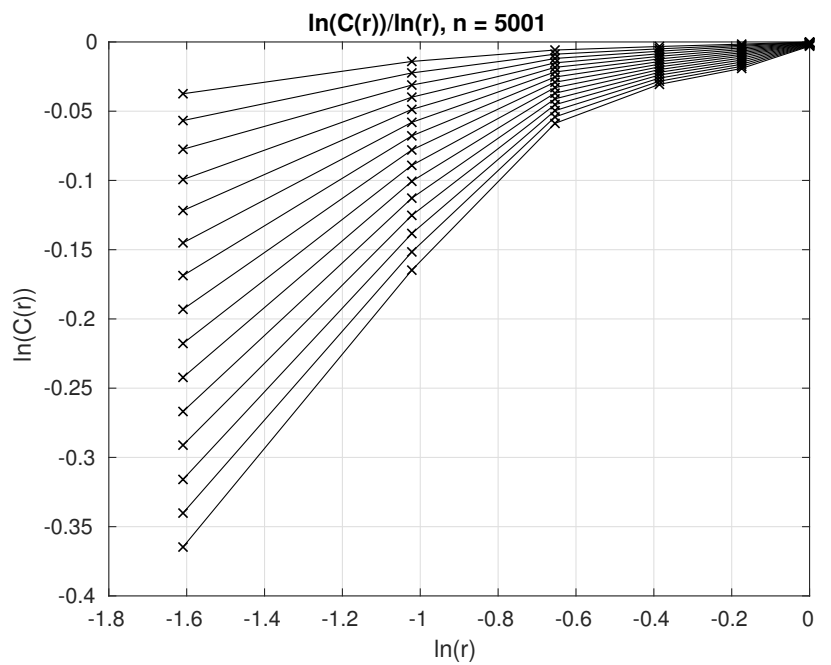

Fig. 3. InterBC89

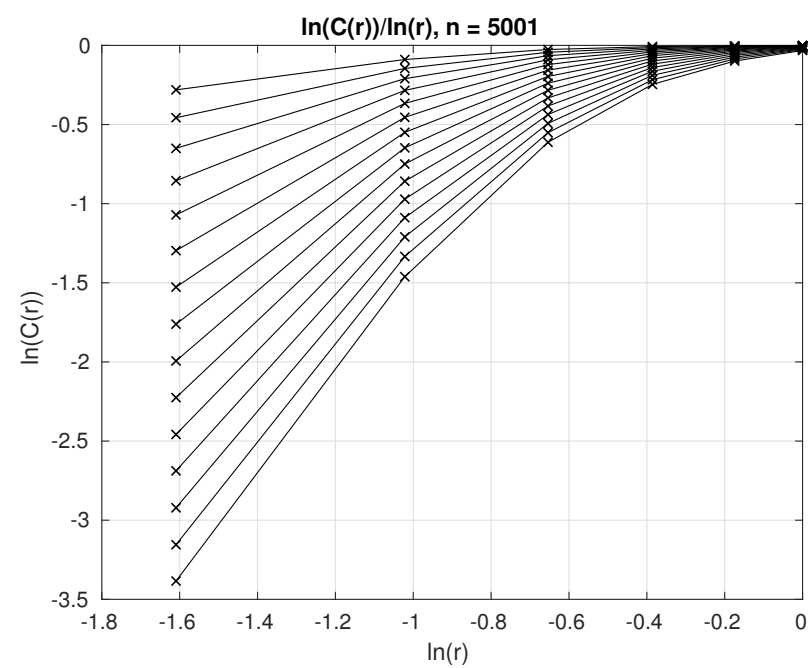

Fig. 4. InterDEC2

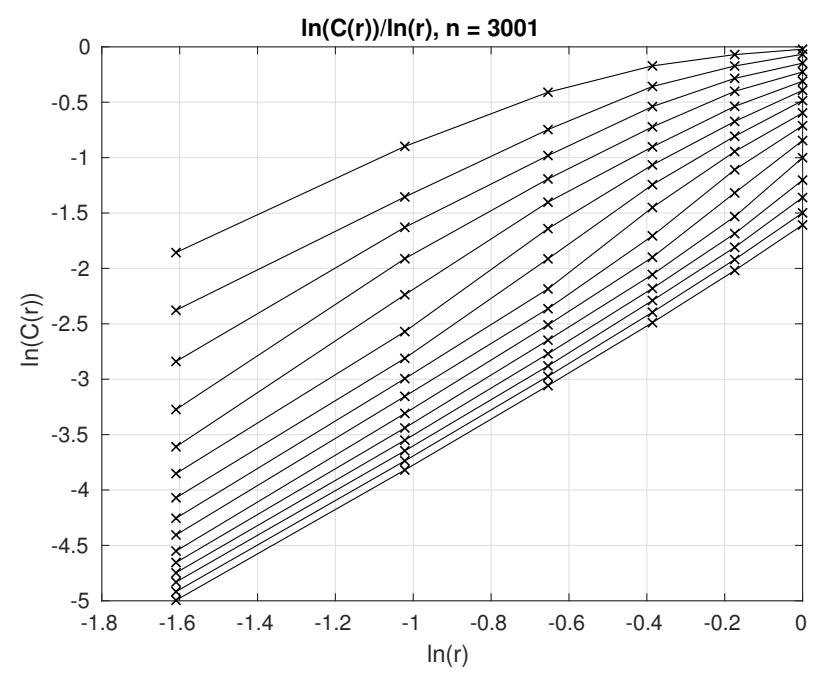

Fig. 5. InterMultipathTCP

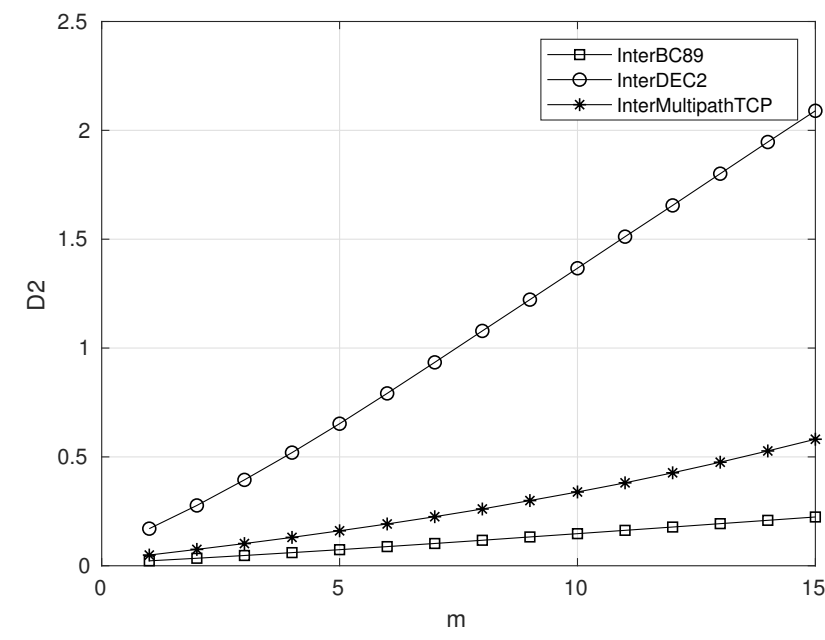

Fig. 6. Dimensão de Correlação $D_{2}$ x Dimensão de Incorporação $m$

à inclinação da reta em sua parcela linear [7]. Essa faixa de valores e todos os $\lambda$ calculados para cada série se encontram organizados na Tabela I.

O parâmetro de Hurst foi calculado através do método descrito em [29]. Como mostrado na Tabela I, as séries InterBC89 e InterDEC2 apresentam dependência de longa duração, característica de séries caóticas autossimilares $(0,5<$ $H<1)$. A série InterMultipathTCP apresenta aleatoriedade, com um $H \approx 0,5$.

\section{TABELA I}

ParÂmetros do Método de Rosenstein, Expoente de Lyapunov $\lambda$ E PARÂMETRo DE HuRst $H$

\begin{tabular}{|l|r|r|r|}
\cline { 2 - 4 } \multicolumn{1}{c|}{} & \multicolumn{1}{c|}{ InterBC89 } & \multicolumn{1}{c|}{ InterDEC2 } & InterMultipathTCP \\
\hline intervalos & $25000: 30000$ & $25000: 30000$ & $25000: 30000$ \\
\hline iterações & 100 & 100 & 100 \\
\hline$\tau$ & 1 & 1 & 1 \\
\hline$D_{2}$ & 0,22 & 2,09 & 0,58 \\
\hline $\mathrm{m}$ & 2 & 6 & 3 \\
\hline tlinear & $1: 2$ & $1: 6$ & $1: 3$ \\
\hline fs & 1 & 1 & 0,1 \\
\hline$\lambda$ & 0,9753 & 0,3824 & 0,0090 \\
\hline $\mathrm{H}$ & 0,6820 & 0,6893 & 0,5685 \\
\hline
\end{tabular}

\section{CONCLUSÕES}

Neste trabalho, o objetivo foi sistematizar um algoritmo de processamento de séries temporais caóticas, provenientes de redes de dados, que otimizam o cálculo de parâmetros de quantificação de sistemas caóticos, ao analisar os valores obtidos de forma a ponderar suas acurácias. Com o método apresentado, informações à respeito das séries temporais foram usadas para reconstruir um espaço de fase equivalente, cujo propósito é permitir o estudo do sistema original de dimensão desconhecida em um espaço de fase análogo e de menor dimensionalidade, reduzindo os gastos computacionais nos cálculos e o tempo necessário.

Foram analisadas séries temporais de tráfego de redes de acordo com a perspectiva da dinâmica não-linear, através da qual foi possível estimar a dimensão de correlação $D_{2}$, com 


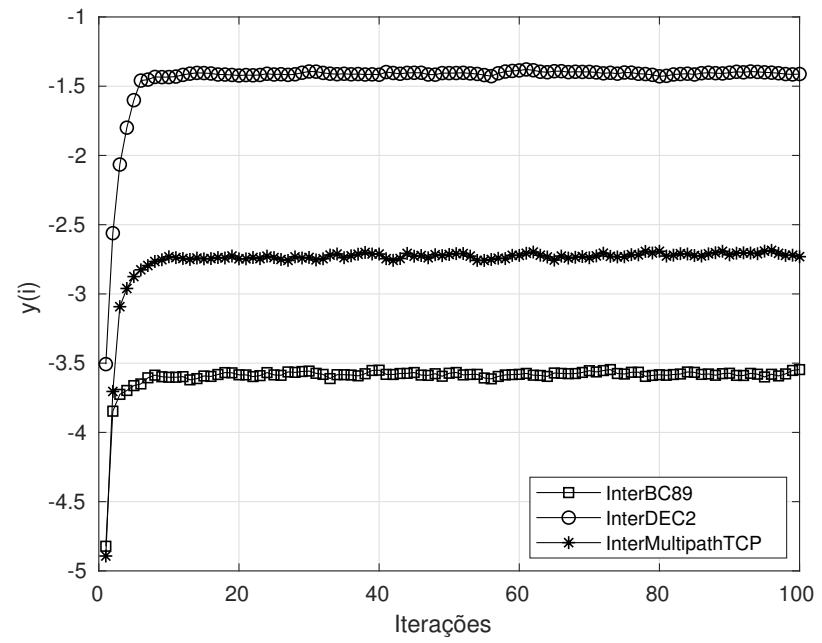

Fig. 7. Região de Escala das Séries Temporais

o uso do algoritmo de Grassberger-Procaccia, a qual apontou um valor fractal (fracionário). O algoritmo Small Data Sets foi usado para calcular o maior expoente de Lyapunov, que retornou um valor positivo, indicando órbitas exponencialmente expansivas. O parâmetro de Hurst confirmou a presença de dependência de longa duração entre as amostras para duas das séries analisadas. Uma delas, a InterMultipathTCP, apresentou um $H \approx 0,5$ mas um $m$ bastante baixo, evidenciando um comportamento misto entre caos (baixa dimensionalidade) e aleatoriedade $(H \approx 0,5)$.

Dessa forma, esse trabalho sintetiza um método de projetar um banco de dados de dimensão desconhecida em um modelo matemático dinâmico e de baixa dimensão, o qual pode ser obtido diretamente dos dados amostrados através da reconstrução do espaço de fase. As características obtidas através desse método podem ser então usadas em procedimentos subsequentes de predição de tráfego, alocação de taxa de transmissão para servidores de rede e processos de medida e manutenção de qualidade de serviço (Quality of Service QoS).

\section{REFERÊNCIAS}

[1] Alves, P. (2019). Chaos in historical prices and volatilities with fivedimensional euclidean spaces. Chaos, Solitons \& Fractals: X, 1:100002.

[2] Alves, P., Duarte, L., and [da Mota], L. (2017). A new characterization of chaos from a time series. Chaos, Solitons \& Fractals, 104:323 - 326

[3] Alves, P., Duarte, L., and [da Mota], L. (2018). Detecting chaos and predicting in dow jones index. Chaos, Solitons \& Fractals, 110:232 238.

[4] Berge, P., Pomeau, Y., and Vidal, C. (1987). Order Within Chaos. Wiley.

[5] Coninck, Q. D., Baerts, M., Hesmans, B., and Bonaventure, O (2016). CRAWDAD dataset uclouvain/mptcp_smartphone (v. 201603-04). Downloaded from https://crawdad.org/uclouvain/ mptcp smartphone/20160304.

[6] Danzig, P., Mogul, J., Paxson, V., and Schwartz, M. (2000). The internet traffic archive datasets. Downloaded from http://ita.ee.lbl. gov/. traceset: combined. Último acesso em 09-22-2010.

[7] Feng, H., Shu, Y., and Yang, O. W. (2009). Nonlinear analysis of wireless lan traffic. Nonlinear Analysis: Real World Applications, 10(2): 1021 - 1028.

[8] Fraser, A. M. and Swinney, H. L. (1986). Independent coordinates for strange attractors from mutual information. Phys. Rev. A, 33:1134-1140.
[9] [Giordano et al. 1997] Giordano, S., Miduri, S., Pagano, M., Russo, F., and Tartarelli, S. (1997). A wavelet-based approach to the estimation of the hurst parameter for self-similar data. In Proceedings of 13th International Conference on Digital Signal Processing, volume 2, pages 479-482. IEEE.

[10] Gleick, J. (1987). Chaos: Making a New Science. Penguin Books, USA

[11] Grassberger, P. and Procaccia, I. (1983a). Characterization of strange attractors.

[12] Grassberger, P. and Procaccia, I. (1983b). Measuring the strangeness of strange attractors. Physica D, 9:189.

[13] Hurst, H. E. (1956). The problem of long-term storage in reservoirs. Hydrological Sciences Journal, 1(3):13-27.

[14] Kantz, H. and Schreiber, T. (2004). Nonlinear time series analysis, volume 7. Cambridge university press.

[15] Kaplan, D. T. and Glass, L. (1993). Coarse-grained embeddings of time series: random walks, gaussian random processes, and deterministic chaos. Physica D: Nonlinear Phenomena, 64(4):431-454.

[16] Leland, W. E., Taqqu, M. S., Willinger, W., and Wilson, D. V. (1994). On the self-similar nature of ethernet traffic (extended version). IEEE/ACM Transactions on Networking, 2(1):1-15.

[17] Liebert, W. and Schuster, H. G. (1989). Proper choice of the time delay for the analysis of chaotic time series. Physics Letters A, 142(2-3):107111.

[18] Liu, Y. and Zhang, J. (2016). Predicting traffic flow in local area networks by the largest lyapunov exponent. Entropy, 18(1):32

[19] Lorenz, E. (1963). Deterministic nonperiodic flow. Journal of Atmospheric Sciences, 20(2):130-148.

[20] Lorenz, E. (1995). The Essence Of Chaos. Jessie and John Danz lectures. Taylor \& Francis.

[21] Mandelbrot, B. B. (1968). Noah, joseph, and operational hydrology. Water resources research. 4 (5) : 909-918; 1968.

[22] Mandelbrot, B. B. (1997). The variation of certain speculative prices In Fractals and scaling in finance, pages 371-418. Springer.

[23] Mandelbrot, B. B. and Hudson, R. L. (2004). The misbehaviour of markets: a fractal view of risk, ruin and reward. Profile, London, UK.

[24] Mañé, R. (1981). On the dimension of the compact invariant sets of certain non-linear maps. Lecture Notes in Mathematics, Berlin Springer Verlag, 898:230.

[25] mirwais (2020). Largest lyapunov exponent with rosenstein's algorithm. MATLAB Central File Exchange. Retrieved February 13, 2020.

[26] Packard, N. H. et al. Geometry from a time series. Phys. Rev. Lett., American Physical Society, v. 45, p. 712-716, Sep 1980.

[27] Prigogine, I. and Stengers, I. (1984). Order Out of Chaos Man's New Dialogue with Nature /Ilya Prigogine and Isabelle Stengers ; Foreword by Alvin Toffler. -. -. Bantam Books, 1984.

[28] Rocha, F. G. C. (2016). Alocação de recursos em redes sem fio ofdm multiusuário utilizando modelagem multifractal adaptativa.

[29] Rocha, F. G. C. and Teles, F. H. V. (2013). Adaptive rate control based on loss probability estimation considering a cascade based multifractal model for the network traffic. IJWMIP, 11.

[30] Rosenstein, M. T., Collins, J. J., and Luca, C. J. D. (1993). A practical method for calculating largest lyapunov exponents from small data sets. Physica D: Nonlinear Phenomena, 65(1):117 - 134.

[31] Silva, M. R. P. d. (2019). Propostas de um modelo para o tráfego de redes de comunicações e de funções de janela para o filtro fir utilizado na tecnologia f-ofdm.

[32] Solis, P. (2007). Uma metodologia de engenharia de tráfego baseada na abordagem auto-similar para a caracterização de parâmetros e a otimização de redes multimídia.

[33] Strogatz, S. (2012). Sync: How Order Emerges From Chaos In the Universe, Nature, and Daily Life. Hyperion.

[34] Strogatz, S. H. (2015). Nonlinear dynamics and chaos: with applications to physics, biology, chemistry, and engineering. CRC Press.

[35] Takens, F. (1981). Detecting strange attractors in turbulence. Lecture Notes in Mathematics, Berlin Springer Verlag, 898:366.

[36] Tempelman, J. R. and Khasawneh, F. A. (2020). A look into chaos detection through topological data analysis. Physica D: Nonlinear Phenomena, 406:132446.

[37] Tian, Z. (2020). Chaotic characteristic analysis of network traffic time series at different time scales. Chaos, Solitons \& Fractals, 130:109412.

[38] Trinh, N. C. and Miki, T. (1999). Dynamic resource allocation for self-similar traffic in atm network. Fifth Asia-Pacific Conference on ... and Fourth Optoelectronics and Communications Conference on Communications, , 1:160-165 vol.1.

[39] Wolf, A., Swift, J. B., Swinney, H. L., and Vastano, J. A. (1985). Determining lyapunov exponents from a time series. Physica D: Nonlinear Phenomena, 16(3):285 - 317. 